\title{
Idelalisib for the treatment of indolent non-Hodgkin lymphoma: a review of its clinical potential
}

\author{
This article was published in the following Dove Press journal: \\ OncoTargets and Therapy \\ 18 May 2016 \\ Number of times this article has been viewed
}

\section{Jacqueline C Barrientos}

CLL Research and Treatment Program, Division of Hematology/Oncology, Department of Medicine, Hofstra Northwell School of Medicine, Hofstra University, Lake Success, NY, USA
Correspondence: Jacqueline $C$ Barrientos CLL Research and Treatment Program, Division of Hematology/ Oncology, Department of Medicine, Hofstra Northwell School of Medicine, Hofstra University, 4I0 Lakeville Road, Lake Success, NY I I042, USA

$\mathrm{Tel}+\mathrm{I} 5164704050$

Fax + I 5164704250

Email jbarrientos@northwell.edu
Abstract: Idelalisib is a first-in-class, oral, selective phosphatidylinositol 3-kinase $\delta$ inhibitor that offers a chemotherapy-free option for patients with relapsed or refractory $(\mathrm{R} / \mathrm{R})$ indolent nonHodgkin lymphoma (iNHL). Clinical trials in iNHL have evaluated idelalisib as monotherapy and as combination therapy with rituximab, bendamustine, and rituximab + bendamustine. When administered to heavily pretreated patients with R/R iNHL, idelalisib monotherapy or combination therapy showed durable antitumor activity accompanied by sustained or improved quality-of-life outcomes. Idelalisib has an acceptable safety profile; however, serious or fatal diarrhea/colitis, hepatoxicity, pneumonitis, and intestinal perforation have occurred in treated patients. Selective inhibition of phosphatidylinositol 3-kinase $\delta$ with idelalisib is a valuable addition to available treatment options for patients with iNHL, many of whom do not respond to or cannot tolerate chemoimmunotherapy. Two Phase III, randomized, placebo-controlled trials of idelalisib as combination therapy with rituximab or bendamustine + rituximab and a Phase I trial of idelalisib in combination with the Bruton's tyrosine kinase inhibitor ONO/GS-4059 in R/R B-cell malignancies are currently ongoing. A Phase III monotherapy trial in previously treated follicular lymphoma or small lymphocytic lymphoma is planned. The development of other kinase inhibitors for the treatment of $\mathrm{NHL}$ raises the potential for new treatment combinations. Additional research is needed to determine optimal therapy (monotherapy vs combination regimens), treatment sequencing, and long-term management.

Keywords: B-cell receptor, follicular lymphoma, elderly, targeted therapy, kinase inhibitor, phosphatidylinositol 3-kinase

\section{Introduction}

Non-Hodgkin lymphomas (NHLs) are a heterogeneous group of solid tumors of the lymphatic system. Approximately $90 \%$ of NHLs arise from B lymphocytes. ${ }^{1}$ The National Cancer Institute estimated that in 2012, 549,625 people in the USA were living with NHL and that in 2015 there will be 71,850 new cases of NHL and 19,790 NHL-related deaths in the USA, which represents $4.3 \%$ of all new cases of cancer and 3.4\% of all cancer-related deaths. ${ }^{2}$ Based on 2010-2012 data, the National Cancer Institute estimated that $2.1 \%$ of men and women will be diagnosed with NHL during their lifetime. ${ }^{2}$

Approximately one third of NHLs are indolent. ${ }^{3,4}$ Indolent NHLs (iNHLs) are slowgrowing tumors with a prolonged natural history, and most patients present with advanced disease. ${ }^{5}$ iNHLs are highly treatable but rarely cured. ${ }^{6}$ Subtypes of iNHL include the most common form, follicular lymphoma (FL), marginal zone lymphoma (MZL), and small lymphocytic lymphoma (SLL), and the least common form, Waldenström macroglobulinemia (WM) or lymphoplasmacytic lymphoma. ${ }^{5,7}$ 
Advanced disease, age $>60-70$ years, male sex, systemic symptoms, poor performance status, tumor burden, absence of interfollicular fibrosis, and cytogenetic abnormalities have been associated with poor prognosis in iNHL. ${ }^{8}$ In elderly patients ( $\geq 90$ years) with lymphoma, identified prognostic factors include high number of medications, anemia, and lymphopenia. ${ }^{9}$ The natural course of FL can vary widely. Over the last decade, there have been significant advances with respect to identifying prognostic markers that can be used to adequately stratify risk in patients with FL. The Follicular Lymphoma International Prognostic Index (FLIPI) includes patient's age, Ann Arbor stage, hemoglobin level, number of nodal sites, and serum lactate dehydrogenase level. ${ }^{10}$ Although it is a widely accepted risk assessment tool, $\sim 20 \%$ of patients with FL have early progression of disease (relapse within 2 years of initial chemoimmunotherapy), and these patients are at increased risk of poor outcomes (5-year overall survival [OS] rate of $50 \%$ vs $90 \%$ in patients without early progression of disease). ${ }^{11}$ Hence, FLIPI score may not be sufficient to identify this high-risk patient subset. Recently, it was demonstrated that integration of the mutational status of seven genes with the FLIPI clinical risk factors improves prognostication for patients with FL receiving first-line immunochemotherapy. ${ }^{12}$ This proposed clinicogenetic risk model (the m7-FLIPI) shows promise in identifying the subset of patients with the highest risk of treatment failure. High-risk patients with FL represent a highly important unmet need for both prognosis and treatment.

Nevertheless, iNHL is most often diagnosed in older individuals. The median age at diagnosis ranges from 65 years for FL to 72 years for MZL, ${ }^{13}$ and the incidence rates increase dramatically with age. ${ }^{7}$ Thus, the growth of the elderly population has likely contributed to the increased rate of NHL diagnoses over time. Elderly patients with iNHL have high rates of medical comorbidities, such as cardiovascular disease, hypertension, and vascular and respiratory tract disorders, ${ }^{14}$ which may limit their ability to tolerate certain myelosuppressive regimens. Retrospective analysis of 133 patients aged $\geq 80$ years with iNHL showed that $74 \%$ had a Cumulative Illness Rating Scale - Geriatric score of $\geq 6 .{ }^{14}$ However, lymphoma is the main cause of death in elderly patients with iNHL, necessitating treatments that combine efficacy with tolerability in a frail patient population. ${ }^{9}$

\section{Current treatment approaches in iNHL}

Though iNHLs remain incurable, treatment is markedly advanced after the advent of the anti-CD20 monoclonal antibody rituximab, which now has widespread use both as monotherapy and in chemoimmunotherapy regimens for first-line and relapsed or refractory $(\mathrm{R} / \mathrm{R})$ diseases. Recommended first-line regimens for grade 1-2 FL include rituximab monotherapy; rituximab, cyclophosphamide, doxorubicin, vincristine, prednisone (R-CHOP); rituximab, cyclophosphamide, vincristine, prednisone (RCVP); and rituximab in combination with bendamustine or lenalidomide. ${ }^{15}$ Recommended second-line and subsequent therapies, in order of preference, are chemoimmunotherapy (same options recommended for first-line treatment), rituximab, lenalidomide \pm rituximab, radioimmunotherapy, idelalisib, fludarabine + rituximab, and rituximab + fludarabine + mitoxantrone + dexamethasone.

Findings from an early trial of rituximab monotherapy in 62 patients with previously untreated iNHL (FL or SLL) demonstrated that 4 weekly infusions of $375 \mathrm{mg} / \mathrm{m}^{2}$ resulted in an objective response rate (ORR) of $47 \%$ at 6 weeks; $45 \%$ of patients had stable disease, and $8 \%$ had tumor progression at that time point. ${ }^{16}$ When the patients with stable disease continued maintenance therapy with the same rituximab regimen administered every 6 months, $73 \%$ achieved ORR and 37\% achieved a complete response (CR). A compelling body of evidence from randomized controlled trials demonstrates that chemoimmunotherapy with rituximab improves outcomes compared with chemotherapy alone in patients with newly diagnosed or relapsed iNHL. ${ }^{17}$ In a randomized trial in patients with previously untreated advanced-stage FL, R-CHOP significantly improved the ORR, OS, and duration of response (DOR) compared with patients randomized to CHOP. ${ }^{18} \mathrm{In}$ a 9-year follow-up study of a Phase II trial of open-label R-CHOP in treatment-naive or previously treated patients with low-grade FL, the median time to progression and DOR were $\sim 7$ years. ${ }^{19}$ Similarly, RCVP has been demonstrated to improve the ORR and OS and extend the time to progression compared with cyclophosphamide, vincristine, and prednisone in patients with previously untreated FL. ${ }^{20,21}$ The combination of bendamustine + rituximab (BR) was more effective than R-CHOP as first-line treatment in patients with iNHL or mantle cell lymphoma and was associated with fewer serious adverse events (AEs; $19 \%$ for BR vs $29 \%$ for R-CHOP). ${ }^{22}$

With advances in treatment, NHL death rates have declined; between 2007 and 2011, rates decreased by $1.8 \%$ per year for men and $2.9 \%$ per year for women, despite a slight increase in NHL incidence rates in men $(0.3 \% /$ year $) .{ }^{23}$ Although the increased response rates and prolonged progression-free survival (PFS) with rituximab monotherapy and combination therapy are encouraging, as noted earlier, $\sim 20 \%$ of patients relapse within 2 years 
of first-line chemoimmunotherapy, ${ }^{24,25}$ and these patients have lower survival rates compared with patients who did not relapse in the first 2 years (OS at 2 years, 68\% vs 97\%, respectively). ${ }^{11}$ Moreover, responses to second-line therapies are often suboptimal, suggesting that salvage regimens are poorly effective after first-line rituximab. ${ }^{26}$

An analysis of data from the National LymphoCare Study revealed that patients older than 80 years were most likely to be treated with first-line rituximab monotherapy or observation only instead of chemoimmunotherapy, and when combination therapy was used, it was generally RCVP. ${ }^{27}$ Although current practice supports the use of rituximabbased regimens in younger, medically fit patients, rituximab monotherapy or watchful waiting is generally recommended for elderly or frail patients. ${ }^{28}$ In the National Comprehensive Cancer Network guidelines, recommendations for first-line therapy in the elderly or infirm are limited to rituximab monotherapy, single-agent alkylators, and radioimmunotherapy. ${ }^{15}$ Taken together, it is clear that there is a substantial unmet need for effective, well-tolerated treatments in $\mathrm{R} / \mathrm{R}$ patients and elderly or frail patients with iNHL.

\section{Idelalisib: a targeted approach to the treatment of iNHL}

The phosphatidylinositol 3-kinases (PI3Ks) are a family of lipid kinases that play an important role in signal transduction and membrane trafficking and are frequently dysregulated in cancers. ${ }^{29,30}$ The class 1 PI3Ks participate in catalytic and regulatory signal transduction and membrane trafficking events. In humans, the PI3K pathway plays an important role in B-cell development, proliferation, migration, adhesion, survival, and immune functions. ${ }^{31}$ Dysregulation of the PI3K pathway seems to confer survival and retention in some malignant B cells. ${ }^{31}$ The PI3K $\delta$ isoform is selectively expressed in leukocytes, making it a desirable therapeutic target for B-cell malignancies. ${ }^{29}$

Idelalisib (Zydelig ${ }^{\circledR}$ ) is a first-in-class, oral, selective, PI3K $\delta$ inhibitor. ${ }^{32}$ In vitro studies have demonstrated that idelalisib potently and selectively inhibits PI $3 \mathrm{~K} \delta$ signaling $^{33}$ and induces apoptosis in patient-derived malignant B cells. ${ }^{34}$ In the USA, idelalisib is approved for the treatment of relapsed FL and relapsed SLL in patients who have received at least two prior systemic therapies and for the treatment of relapsed chronic lymphocytic leukemia (CLL) in combination with rituximab in patients for whom rituximab alone would be considered appropriate therapy because of other comorbidities. ${ }^{35}$ In Europe, idelalisib is approved as monotherapy for adults with refractory FL and in combination with rituximab for adults with CLL. ${ }^{36}$ Idelalisib represents a rational treatment option for patients who are not ideal candidates for standard chemotherapy. The high degree of leukocyte selectivity of idelalisib forms the basis for its clinical activity and acceptable toxicity profile.

\section{Clinical efficacy of idelalisib in iNHL}

The NCCN Clinical Practice Guidelines in Oncology include idelalisib as a recommended treatment option in second-line or subsequent therapy in patients with grade 1-2 FL, making it one of the few alternative treatments available for patients with iNHL that is refractory to rituximab or alkylating agents. ${ }^{15}$ The efficacy of idelalisib monotherapy was first reported in a Phase I dose-ranging study of 64 patients with previously treated iNHL (FL, $n=38$; SLL, $n=11$; MZL, $n=6$; lymphoplasmacytic lymphoma, $n=9$; Table 1). ${ }^{37}$ The patients had received a median of 4 (range: $1-10$ ) prior therapies; $>90 \%$ had previously received rituximab or an alkylating

Table I Efficacy of idelalisib in clinical trials in iNHL

\begin{tabular}{|c|c|c|c|}
\hline Study & Regimen & Population & Efficacy results \\
\hline Phase I dose-ranging study ${ }^{37}$ & $\begin{array}{l}\text { IDELA 50-350 mg QD or BID } \\
\text { continuously or intermittently } \\
\text { ( } 3 \text { weeks on, I week off) }\end{array}$ & $\begin{array}{l}\mathrm{N}=64 \mathrm{R} / \mathrm{R} \text { iNHL; } 97 \% \text { had } \\
\text { rituximab as prior therapy }\end{array}$ & $\begin{array}{l}\text { ORR, } 47 \% \\
\text { TTR, I.3 mo } \\
\text { DOR, } 18.4 \mathrm{mo} \\
\text { PFS, } 7.6 \mathrm{mo}\end{array}$ \\
\hline Phase I combination therapy study ${ }^{38}$ & $\begin{array}{l}\text { IDELA I } 50 \mathrm{mg} \text { BID + rituximab, } \\
\mathrm{B} \text {, or both }\end{array}$ & $\begin{array}{l}\mathrm{N}=79 \mathrm{R} / \mathrm{R} \text { iNHL; } 58 \% \\
\text { were refractory to } \\
\text { rituximab }\end{array}$ & $\begin{array}{l}\text { ORR, } 81 \% \\
\text { CR, } 33 \% \\
\text { ORR for IDELA + R (75\%), IDELA + B } \\
\text { (88\%), and IDELA + R + B (79\%) } \\
\text { DOR for IDELA + R ( } 28.6 \text { mo); not } \\
\text { reached for IDELA + B or IDELA + R + B }\end{array}$ \\
\hline Phase II monotherapy study ${ }^{3}$ & IDELA I50 mg BID monotherapy & $\begin{array}{l}\mathrm{N}=125 \mathrm{iNHL} \text { refractory } \\
\text { to rituximab and an } \\
\text { alkylating agent }\end{array}$ & $\begin{array}{l}\text { ORR, } 57 \% \\
\text { TTR, } 1.9 \mathrm{mo} \\
\text { DOR, } 12.5 \mathrm{mo} \\
\text { PFS, II mo }\end{array}$ \\
\hline
\end{tabular}

Abbreviations: B, bendamustine; BID, twice daily; CR, complete response; DOR, duration of response; IDELA, idelalisib; iNHL, indolent non-Hodgkin lymphoma; mo, months; ORR, objective response rate; PFS, progression-free survival; QD, once daily; R, rituximab; R/R, relapsed or refractory; TTR, time to response. 
agent, and $58 \%$ were refractory to the last prior therapy. Idelalisib dose ranging consisted of continuous regimens of $50-350 \mathrm{mg}$ once or twice daily or an intermittent regimen of $150 \mathrm{mg}$ twice daily (3 weeks on, 1 week off every 4 weeks). Nineteen patients $(30 \%)$ completed the initial 48 -week study and entered an extension phase. The mean treatment duration was 9.3 months, and the maximum tolerated dose was not identified at study end point. The ORR was $47 \%$ $(n=30)$ in the total study population and 59\% in patients treated with continuous higher doses. Responding patients had a rapid reduction in lymphadenopathy with a median time to response of 1.3 months (range: 0.7-14). The median (range) DOR and PFS were 18.4 (0.03-34) months and 7.6 (0.03-37) months, respectively. Because the median PFS was longer in patients treated with higher dose continuous therapy (16.8 months; range: $1-37$ months) than in patients receiving lower doses or intermittent therapy (3.7 months; range: $0.5-33$ months), idelalisib doses of $\geq 150 \mathrm{mg}$ twice daily were identified for further study.

Idelalisib + rituximab or bendamustine or both were evaluated in a Phase I study of 79 patients with R/R iNHL (Table 1). ${ }^{38}$ The patients had received a median of 3 (range: 1-11) prior courses of therapy. Most patients had been treated with rituximab (98\%) or an alkylating agent (86\%), and 58\% were refractory to rituximab. The first eight patients in each group received idelalisib $100 \mathrm{mg}$ twice daily; thereafter, patients were treated with $150 \mathrm{mg}$ twice daily. Rituximab was administered as a $375 \mathrm{mg} / \mathrm{m}^{2}$ infusion once weekly for eight doses, and the bendamustine dose was $90 \mathrm{mg} / \mathrm{m}^{2}$ administered on days 1 and 2 of each cycle for six cycles. The ORR was $81 \%$, and $33 \%$ of patients achieved a CR. The ORRs for the idelalisib + rituximab, idelalisib + bendamustine, and idelalisib + BR groups were 75\%, 88\%, and 79\%, respectively. The median DOR for idelalisib + rituximab-treated patients was 28.6 months, but at the time these data were presented, the DOR had not yet been reached for the other treatment groups. A durable response of over 2 years supported further evaluation of idelalisib combination regimens in Phase III studies.

Based on the promising results from the Phase I studies, a Phase II trial of idelalisib monotherapy (150 mg twice daily) was conducted in 125 heavily pretreated patients with relapsed iNHL (Table 1). ${ }^{3}$ The patients had received a median of 4 (range: 2-12) prior treatment courses; 100\% were refractory to rituximab, $99 \%$ to alkylating agents, and $75 \%$ to bendamustine. FL was the most common subtype (58\%), and $17 \%$ of patients with FL had grade $3 \mathrm{~A}$ disease. With a median duration of idelalisib treatment of 6.6 months (range:
0.6-23.9 months), the ORR was 57\% $(\mathrm{n}=71)$ and responses were rapid (median time to response, 1.9 months [range: 1.6-8.3 months]) and durable (median DOR, 12.5 months [range: 0.03-14.8 months]). The ORR was similar in subgroups stratified by age $(57 \%$ in patients aged $<65$ years and $\geq 65$ years), presence of bulky disease (no [57\%] vs yes $[58 \%])$, number of previous therapies $(<4[50 \%]$ and $\geq 4$ [62\%]), refractoriness to last prior therapy (no [69\%] vs yes [55\%]), and refractoriness to bendamustine (no [50\%] vs yes [59\%]). The median (range) PFS was 11.0 (0.03-16.6) months, and at data cutoff, the median (range) OS was 20.3 (0.7-22.0) months.

Patients in this study demonstrated clinically meaningful improvements in health-related quality-of-life metrics, including lymphoma-related concerns, based on the Functional Assessment of Cancer Therapy - Lymphoma. ${ }^{39}$ A decision-analysis model that extracted survival data and compared them with health outcomes data from a claims analysis database showed that idelalisib therapy was associated with benefits in life-years gained (7.8 months), progression-free life-years gained (4.6 months), and qualityadjusted life-years gained ( 0.5 year). ${ }^{40}$

A subsequent subgroup analysis evaluated idelalisib efficacy and safety in patients with FL $(n=72)$ enrolled in the Phase II trial, with a median (range) treatment duration of 6.5 (0.6-31.0) months and median (range) follow-up of 19.4 (0.7-35.6) months. ${ }^{41}$ In these R/R patients with FL (54\% had a high FLIPI risk score at baseline; 79\% were refractory to at least two regimens; $17 \%$ had FL grade $3 \mathrm{~A}$ ), the ORR was $55.6 \%$ and median (range) PFS was 11.0 (0-30.6) months versus 5.1 (4.4-6.0) months with the last prior therapy. The median OS was not reached, and OS rates at 12 months, 18 months, and 24 months were $88.1 \%, 74.2 \%$, and $69.8 \%$, respectively. These data suggest that patients with high-risk FL may benefit from a targeted therapy such as idelalisib.

\section{Safety and tolerability in iNHL}

Idelalisib exhibited an acceptable safety profile in patients treated for up to 4 years in the Phase I and II trials. ${ }^{3,37,38}$ In the Phase II trial, ${ }^{3}$ the most frequent AEs ( $\geq 20 \%$ ) were diarrhea $(43 \%)$, nausea $(30 \%)$, fatigue $(30 \%)$, cough $(29 \%)$, and pyrexia (28\%). Twenty-five patients discontinued because of AEs, including elevated liver transaminases, pneumonia/ pneumonitis, diarrhea, colitis, and neutropenia. Grade $\geq 3$ treatment-emergent AEs occurring in $>2 \%$ of patients were diarrhea (13\%), pneumonia (7\%), and dyspnea $(3 \%)$. Increased bleeding or bruising was not reported. ${ }^{42}$ Grade $\geq 3$ 
diarrhea and/or colitis occurred in $16 \%$ of patients, with a median onset of 6 months; six cases resolved spontaneously or after dose reduction and eight cases resolved with temporary interruption of therapy. Idelalisib was permanently discontinued in six patients with grade $\geq 3$ diarrhea. Laboratory abnormalities occurring in $\geq 10 \%$ of patients included neutropenia (any grade, 56\%; grade $\geq 3,27 \%$ ), alanine aminotransferase (ALT) elevation (any grade, $47 \%$; grade $\geq 3,13 \%$ ), aspartate aminotransferase (AST) elevation (any grade, $35 \%$; grade $\geq 3,8 \%$ ), anemia (any grade, $28 \%$; grade $\geq 3,2 \%$ ), thrombocytopenia (any grade, $26 \%$; grade $\geq 3,6 \%$ ), alkaline phosphatase elevation (any grade, $22 \%$; grade $\geq 3,0 \%$ ), and bilirubin elevation (any grade, $10 \%$; grade $\geq 3,0 \%$ ). The median onset for grade $\geq 3$ liver transaminase elevations was 6.3 weeks after starting idelalisib $(n=16)$; all such events were reversible, with a median onset of resolution to grade $\leq 1$ of 3.9 weeks.

Idelalisib prescribing information includes a black box warning for fatal and severe hepatotoxicity, diarrhea or colitis, pneumonitis, and intestinal perforation. ${ }^{35}$ Patients should be monitored for the development of events, and treatment should be reduced or discontinued if they occur, as per the prescribing information. Idelalisib should be discontinued if intestinal perforation is suspected. An expert panel of hematologists and one gastroenterologist has provided further guidance for the management of idelalisib treatment-emergent diarrhea/colitis. ${ }^{43}$ The panel unanimously concluded that diarrhea most likely to be related to idelalisib treatment typically has a late onset (after 2 months) and responds poorly to antibiotics or antidiarrheal medications. ${ }^{43}$ Based on anecdotal effectiveness, the panel recommended that once infectious source has been ruled out, budesonide or steroid (oral or intravenous) therapy should be initiated and continued until diarrhea resolves. In our clinical experience, diarrhea can occur several months after idelalisib initiation; hence, it is important to remind patients to alert their medical providers of any diarrhea lasting $>24$ hours.

\section{Future considerations}

There are currently six ongoing clinical studies of idelalisib in iNHL registered on the ClinicalTrials.gov web site (Table 2). These include two Phase III trials evaluating idelalisib in combination with rituximab (NCT01732913; Yosemite) and in combination with BR (NCT01732926; Bridalveil) in previously treated patients with iNHL. The Yosemite trial began enrolling patients in January 2013 and is expected to be completed in June 2022 (primary data collection will be completed in June 2019). The Bridalveil trial began in January 2013 with a planned completion date of September 2022 (September 2017 for completion of primary data collection). A third Phase III trial is evaluating the effectiveness and safety of idelalisib monotherapy in previously treated FL or SLL (NCT02536300), and a Phase II trial is evaluating the effectiveness and safety of idelalisib in previously treated WM (NCT02439138). Also currently ongoing are a Phase I trial evaluating the treatment combination of idelalisib with the investigational Bruton's tyrosine kinase (BTK) inhibitor ONO/GS-4059 (NCT02457598) in R/R patients and a Phase I/II trial of idelalisib monotherapy in previously treated iNHL (NCT01306643).

Clinical trials are needed to evaluate the role of idelalisib as the first-line therapy in iNHL, address the sequencing of idelalisib with respect to chemotherapy, and determine optimal combination regimens. At present, idelalisib is

Table 2 Ongoing or planned idelalisib trials in iNHL

\begin{tabular}{|c|c|c|c|}
\hline Study & Regimen & Population & $\begin{array}{l}\text { Target accrual } \\
\text { (status)/primary } \\
\text { end point(s) }\end{array}$ \\
\hline Phase I (NCT02457598) & Idelalisib + ONO/GS-4059; open label & $\begin{array}{l}\text { Relapsed or refractory B-cell } \\
\text { malignancies }\end{array}$ & $\begin{array}{l}\mathrm{N}=138 \text { (recruiting) } \\
\text { Safety, toxicity, ORR }\end{array}$ \\
\hline Phase I/II (NCT0I306643) & Idelalisib monotherapy; open label & Previously treated iNHL & $\begin{array}{l}\mathrm{N}=15 \text { (recruiting) } \\
\text { Safety, response rate }\end{array}$ \\
\hline Phase II (NCT02439I38) & Idelalisib monotherapy; open label & $\begin{array}{l}\text { Previously treated Waldenström } \\
\text { macroglobulinemia }\end{array}$ & $\begin{array}{l}\mathrm{N}=30 \text { (recruiting) } \\
\text { ORR }\end{array}$ \\
\hline Phase III (NCTOI7329I3; Yosemite) & $\begin{array}{l}\text { Idelalisib + rituximab; placebo } \\
\text { controlled }\end{array}$ & Previously treated iNHL & $\begin{array}{l}\mathrm{N}=375 \text { (recruiting) } \\
\mathrm{PFS} \text {, response rate, OS }\end{array}$ \\
\hline Phase III (NCT0I732926; Bridalveil) & $\begin{array}{l}\text { Idelalisib + bendamustine + rituximab; } \\
\text { placebo controlled }\end{array}$ & Previously treated iNHL & $\begin{array}{l}\mathrm{N}=450 \text { (recruiting) } \\
\text { PFS }\end{array}$ \\
\hline Phase III (NCT02536300) & Idelalisib monotherapy & $\begin{array}{l}\text { Previously treated follicular lymphoma } \\
\text { or small lymphocytic lymphoma }\end{array}$ & $\begin{array}{l}\mathrm{N}=240 \text { (recruiting) } \\
\text { Safety, ORR }\end{array}$ \\
\hline
\end{tabular}

Abbreviations: iNHL, indolent non-Hodgkin lymphoma; ORR, overall response rate; OS, overall survival; PFS, progression-free survival. 
approved in the USA for the management of relapsed FL or SLL in patients who have received at least two prior systemic therapies. ${ }^{35}$ In fit patients, there are no data to guide clinicians in deciding between initiating chemotherapy, which can cause myelosuppression and other complications, or initiating a targeted therapy such as idelalisib that could avoid myelosuppressive complications. The limited data available on idelalisib as frontline therapy in CLL (a different B-cell lymphoproliferative disorder) seem to suggest that it may be associated with an increased risk of severe immunemediated toxicities in this setting. ${ }^{44,45}$ There were high rates of severe toxicities in patients with CLL receiving frontline therapy with idelalisib + ofatumumab (grade $\geq 3$ toxicity, $76 \%)^{44}$ or idelalisib + rituximab (grade $\geq 3$ toxicity, $89 \%$ ). ${ }^{45}$ The most common grade $\geq 3$ toxicities were ALT/AST elevation $(57 \%)$, enterocolitis (14\%), and pneumonitis (10\%) in the idelalisib + ofatumumab study and diarrhea or colitis $(42 \%)$ and pneumonia (19\%) in the idelalisib + rituximab study. In addition to further clinical trials, preclinical studies may be useful to identify the mechanisms for such toxicity and markers for selecting patients who tolerate frontline idelalisib well. ${ }^{44}$

Additional unanswered questions relate to long-term outcomes on continued idelalisib therapy. Current practice is to continue idelalisib treatment until disease progression or the occurrence of unacceptable toxicity, but little is known about whether treatment can be safely discontinued in patients who have achieved a clinical end point. Moreover, to guide treatment in patients who stop responding to idelalisib, clinical and preclinical data are needed to elucidate idelalisib resistance and to identify effective salvage therapy, which could involve treatment add-on or switching to a different targeted agent. The possible benefit of idelalisib beyond disease progression is not known because all clinical trials conducted to date have excluded patients who demonstrated disease progression while on therapy.

The development and clinical investigation of other targeted therapies create the potential for many novel idelalisib treatment combinations for iNHL. In addition to the BTK inhibitor ibrutinib (Imbruvica ${ }^{\circledR}$ ), which is approved for use in MCL, CLL, and WM, ${ }^{46}$ a number of other kinase inhibitors are currently in development (Table 3 ). These include the PI3K $\delta$ inhibitors TGR-1202 and AMG 319, the PI3K $\delta \gamma$ inhibitor duvelisib (IPI-145), the PI3K $\alpha \delta$ inhibitor copanlisib (BAY 80-6946), and the B-cell lymphoma-2 inhibitor venetoclax (ABT-199). Duvelisib and copanlisib are currently in Phase III development in FL and NHL, respectively. A Phase II study of duvelisib monotherapy in 32 patients
Table 3 Novel targeted therapies in development for iNHL

\begin{tabular}{ll}
\hline Agent (development phase in iNHL) & Therapeutic target \\
\hline TGR-I 202 (Phase I) & PI3K $\delta$ \\
AMG 3 I9 (Phase I) & PI3K $\delta$ \\
IPI-I 45/durelisib (Phase III) & PI3K $\delta$ and PI3K $\gamma$ \\
BAY 80-6946/copanlisib (Phase III) & PI3K $\alpha$ and PI3K $\delta$ \\
ABT-I 99/venetoclax (Phase II) & BCL-2 \\
ONO/GS-4059 (Phase I) & BTK \\
ACP-I 96/acalabrutinib (Phase I/II) & BTK \\
\hline
\end{tabular}

Abbreviations: BCL-2, B-cell lymphoma 2; BTK, Bruton's tyrosine kinase; $\mathrm{NHL}$, indolent non-Hodgkin lymphoma; PI3K, phosphatidylinositol 3-kinase.

with $\mathrm{R} / \mathrm{R}$ iNHL demonstrated clinical activity at doses of $15 \mathrm{mg}$ twice daily to $75 \mathrm{mg}$ twice daily with an ORR of $65 \%$, including five CRs, 14 partial responses, and one minor response. ${ }^{47}$ The most common grade $\geq 3$ AEs were ALT/AST increase ( $41 \%$ ) and diarrhea (22\%) and grade $\geq 3$ neutropenia was reported in $31 \%$ of patients. In a Phase IIA study in 33 patients with $\mathrm{R} / \mathrm{R}$ iNHL or CLL, copanlisib monotherapy produced an ORR of $47 \%$, with one $\mathrm{CR}$, one unconfirmed CR, and 13 partial responses. ${ }^{48}$ The most common grade $\geq 3$ AEs were hypertension (49\%), neutropenia (30\%), hyperglycemia (30\%), and anemia (15\%). Two second-generation BTK inhibitors, ONO-4059 (Phase I) and acalabrutinib (ACP-196; Phase I/II), are currently in development.

With the abundance of potential combination therapies, it is important to determine in a clinical trial setting whether such strategies can improve outcomes without increasing toxicity compared with idelalisib monotherapy. A Phase II clinical trial of idelalisib in combination with the spleen tyrosine kinase inhibitor entospletinib in $\mathrm{R} / \mathrm{R}$ CLL or NHL was discontinued due to frequent occurrence of pneumonitis (NCT01796470). ${ }^{49}$ Two trials evaluating idelalisib + lenalidomide in recurrent FL (NCT01644799) and lenalidomide \pm idelalisib in mantle cell lymphoma (NCT01838434) were suspended owing to tumor flares in treated patients. Moreover, the triplet of idelalisib + lenalidomide + rituximab has been associated with unexpected and serious toxicities, including rash, fevers, hypotension, and hepatotoxicity, which were thought to be related to high-level immune activation. ${ }^{50,51}$ In contrast, results of a prespecified interim analysis of a Phase III, randomized, double-blind trial comparing idelalisib + BR with placebo + BR in patients with R/R CLL show superior PFS (primary end point) and OS (secondary end point) in the idelalisib versus placebo arm, without increased toxicity compared with previous studies. ${ }^{52}$ However, that study did not address whether the addition of bendamustine adds substantially to the backbone of idelalisib and rituximab or idelalisib 
monotherapy in CLL. It is for this reason that well-designed clinical trials are essential to identify effective treatment strategies that can improve response and remission duration while maintaining an acceptable toxicity profile.

\section{Conclusion}

The iNHLs are a heterogeneous group of malignancies that are treatable but not curable. The anti-CD20 antibody rituximab has been the mainstay of first- and second-line treatments of iNHL as monotherapy or chemoimmunotherapy. Unfortunately, initial reductions in tumor burden following rituximab therapy often are not sustained, and response rates decline with repeated courses. Patients with iNHL often endure multiple courses of chemoimmunotherapy that increase the risk of serious cardiac toxicity and myelosuppression. This is particularly problematic in elderly patients with iNHL.

Selective inhibition of the PI3K $\delta$ pathway with oral idelalisib represents a viable and clinically useful chemotherapyfree option for patients with iNHL. Clinical trial data demonstrate the effectiveness of idelalisib as monotherapy or combination therapy in patients with $\mathrm{R} / \mathrm{R}$ iNHL and as monotherapy in high-risk FL, with an acceptable AE profile. Ongoing trials will provide additional Phase III data and inform new treatment combinations. An important objective for future research is to determine whether combination therapies can succeed in improving outcomes over monotherapy without increasing toxicity.

\section{Acknowledgments}

Jacqueline C Barrientos' work is supported in part by the National Institutes of Health/National Center for Advancing Translational Sciences Grant UL1TR00457, the 2015 American Society of Hematology-Harold Amos Medical Faculty Development Program fellowship, and philanthropic contributions from the Nash Family Foundation, Karches Family Foundation, Jerome Levy Foundation, Leon Levy Foundation, and Frank and Mildred Feinberg Foundation. Editorial assistance for the development of this manuscript was provided by Sally Laden, MS, a medical writer at C4 MedSolutions, LLC (Yardley, PA, USA), a CHC Group company, who received funding from Gilead Sciences, Inc. Gilead did not contribute to, review, edit, or comment on the paper.

\section{Disclosure}

Dr Barrientos reports the following disclosures: institutional research funding from Gilead and AbbVie and advisory board consulting for Gilead, AbbVie, Jannsen, and
Genentech. The author reports no other conflicts of interest in this work.

\section{References}

1. Shankland KR, Armitage JO, Hancock BW. Non-Hodgkin lymphoma. Lancet. 2012;380(9844):848-857.

2. SEER. SEER Stat Fact Sheets: Non-Hodgkin Lymphoma. Bethesda, MD: National Cancer Institute; 2012.

3. Gopal AK, Kahl BS, de Vos S, et al. PI3K $\delta$ inhibition by idelalisib in patients with relapsed indolent lymphoma. NEngl J Med. 2014;370(11): 1008-1018.

4. Morton LM, Wang SS, Devesa SS, Hartge P, Weisenburger DD, Linet MS. Lymphoma incidence patterns by WHO subtype in the United States, 1992-2001. Blood. 2006;107(1):265-276.

5. Lunning MA, Vose JM. Management of indolent lymphoma: where are we now and where are we going. Blood Rev. 2012;26(6):279-288.

6. Jaffe ES. Relationship of classification to biologic behavior of nonHodgkin's lymphomas. Semin Oncol. 1986;13(4 suppl 5):3-9.

7. Howlader N, Noone AM, Krapcho M, et al [webpage on the Internet] SEER Cancer Statistics Review, 1975-2012; 2015. Available from: http://seer.cancer.gov/csr/1975_2012/. Accessed October 6, 2015.

8. Horning SJ. Natural history of and therapy for the indolent nonHodgkin's lymphomas. Semin Oncol. 1993;20(5 suppl 5):75-88.

9. Trebouet A, Marchand T, Lemal R, et al. Lymphoma occurring in patients over 90 years of age: characteristics, outcomes, and prognostic factors. A retrospective analysis of 234 cases from the LYSA. Ann Oncol. 2013;24(10):2612-2618.

10. Solal-Celigny P, Roy P, Colombat P, et al. Follicular lymphoma international prognostic index. Blood. 2004;104(5):1258-1265.

11. Casulo C, Byrtek M, Dawson KL, et al. Early relapse of follicular lymphoma after rituximab plus cyclophosphamide, doxorubicin, vincristine, and prednisone defines patients at high risk for death: an analysis from the National LymphoCare Study. J Clin Oncol. 2015;33(23): 2516-2522.

12. Pastore A, Jurinovic V, Kridel R, et al. Integration of gene mutations in risk prognostication for patients receiving first-line immunochemotherapy for follicular lymphoma: a retrospective analysis of a prospective clinical trial and validation in a population-based registry. Lancet Oncol. 2015;16(9):1111-1122.

13. Smith A, Howell D, Patmore R, Jack A, Roman E. Incidence of haematological malignancy by sub-type: a report from the Haematological Malignancy Research Network. Br J Cancer. 2011;105(11):1684-1692.

14. Nabhan C, Smith SM, Helenowski I, et al. Analysis of very elderly ( $\geq 80$ years) non-Hodgkin lymphoma: impact of functional status and co-morbidities on outcome. Br J Haematol. 2012;156(2):196-204.

15. National Comprehensive Cancer Network [webpage on the Internet]. NCCN Clinical Practice Guidelines in Oncology: Non-Hodgkin's Lymphomas (version 1.2015); 2015. Available from: http://www.nccn.org/ professionals/physician_gls/f_guidelines.asp\#site. Accessed October 6, 2015.

16. Hainsworth JD. Rituximab as first-line and maintenance therapy for patients with indolent non-Hodgkin's lymphoma: interim follow-up of a multicenter phase II trial. Semin Oncol. 2002;29(1 suppl 2): 25-29.

17. Schulz H, Bohlius JF, Trelle S, et al. Immunochemotherapy with rituximab and overall survival in patients with indolent or mantle cell lymphoma: a systematic review and meta-analysis. J Natl Cancer Inst. 2007;99(9):706-714.

18. Hiddemann W, Kneba M, Dreyling M, et al. Frontline therapy with rituximab added to the combination of cyclophosphamide, doxorubicin, vincristine, and prednisone (CHOP) significantly improves the outcome for patients with advanced-stage follicular lymphoma compared with therapy with CHOP alone: results of a prospective randomized study of the German Low-Grade Lymphoma Study Group. Blood. 2005; 106(12):3725-3732. 
19. Czuczman MS, Weaver R, Alkuzweny B, Berlfein J, Grillo-Lopez AJ. Prolonged clinical and molecular remission in patients with low-grade or follicular non-Hodgkin's lymphoma treated with rituximab plus CHOP chemotherapy: 9-year follow-up. J Clin Oncol. 2004;22(23): $4711-4716$

20. Marcus R, Imrie K, Belch A, et al. CVP chemotherapy plus rituximab compared with CVP as first-line treatment for advanced follicular lymphoma. Blood. 2005;105(4):1417-1423.

21. Marcus R, Imrie K, Solal-Celigny P, et al. Phase III study of R-CVP compared with cyclophosphamide, vincristine, and prednisone alone in patients with previously untreated advanced follicular lymphoma. J Clin Oncol. 2008;26(28):4579-4586.

22. Rummel MJ, Niederle N, Maschmeyer G, et al. Bendamustine plus rituximab versus CHOP plus rituximab as first-line treatment for patients with indolent and mantle-cell lymphomas: an open-label, multicentre, randomised, phase 3 non-inferiority trial. Lancet. 2013; 381(9873):1203-1210.

23. American Cancer Society [webpage on the Internet]. Cancer Facts \& Figures 2015. Available from: http://www.cancer.org/acs/groups/content/@editorial/documents/document/acspc-044552.pdf. Accessed March 31, 2015.

24. Casulo C, Burack WR, Friedberg JW. Transformed follicular nonHodgkin lymphoma. Blood. 2015;125(1):40-47.

25. Press OW, Unger JM, Rimsza LM, et al. Phase III randomized intergroup trial of CHOP plus rituximab compared with $\mathrm{CHOP}$ chemotherapy plus (131)iodine-tositumomab for previously untreated follicular non-Hodgkin lymphoma: SWOG S0016. J Clin Oncol. 2013;31(3): 314-320.

26. Chao MP. Treatment challenges in the management of relapsed or refractory non-Hodgkin's lymphoma - novel and emerging therapies. Cancer Manage Res. 2013;5:251-269.

27. Nabhan C, Byrtek M, Rai A, et al. Disease characteristics, treatment patterns, prognosis, outcomes and lymphoma-related mortality in elderly follicular lymphoma in the United States. Br J Haematol. 2015; 170(1):85-95.

28. Hiddemann $\mathrm{W}$, Cheson BD. How we manage follicular lymphoma. Leukemia. 2014;28(7):1388-1395.

29. Vanhaesebroeck B, Welham MJ, Kotani K, et al. P110delta, a novel phosphoinositide 3-kinase in leukocytes. Proc Natl Acad Sci US A. 1997; 94(9):4330-4335

30. Vanhaesebroeck B, Guillermet-Guibert J, Graupera M, Bilanges B. The emerging mechanisms of isoform-specific PI3K signalling. Nat Rev Mol Cell Biol. 2010;11(5):329-341.

31. Pauls SD, Lafarge ST, Landego I, Zhang T, Marshall AJ. The phosphoinositide 3-kinase signaling pathway in normal and malignant $\mathrm{B}$ cells: activation mechanisms, regulation and impact on cellular functions. Front Immunol. 2012;3:224

32. Forcello N, Saraiya N. Idelalisib: the first-in-class phosphatidylinositol 3-kinase inhibitor for relapsed CLL, SLL, and indolent NHL. $J A d v$ Pract Oncol. 2014;5:455-459.

33. Somoza JR, Koditek D, Villasenor AG, et al. Structural, biochemical, and biophysical characterization of idelalisib binding to phosphoinositide 3-kinase delta. J Biol Chem. 2015;290(13):8439-8446.

34. Lannutti BJ, Meadows SA, Herman SE, et al. CAL-101, a p110delta selective phosphatidylinositol-3-kinase inhibitor for the treatment of B-cell malignancies, inhibits PI3K signaling and cellular viability. Blood. 2011;117(2):591-594.

35. ZYDELIG ${ }^{\circledR}$ (idelalisib tablets) [full prescribing information]. Foster City, CA: Gilead Sciences, Inc.; 2014.

36. European Medicines Agency [webpage on the Internet]. Idelalisib. Summary of Product Characteristics; 2014. Available from: https:// www.medicines.org.uk/emc/medicine/29202. Accessed October 6, 2015.
37. Flinn IW, Kahl BS, Leonard JP, et al. Idelalisib, a selective inhibitor of phosphatidylinositol 3-kinase-delta, as therapy for previously treated indolent non-Hodgkin lymphoma. Blood. 2014;123(22): 3406-3413.

38. de Vos S, Wagner-Johnston ND, Coutre SE, et al. Durable responses following treatment with the PI3K-delta inhibitor idelalisib in combination with rituximab, bendamustine, or both, in recurrent indolent non-Hodgkin lymphoma: phase I/II results. Blood. 2014;124(21): abstract3063.

39. Wagner-Johnston ND, Gopal AK, Kahl BS, et al. Patient-reported outcomes data from a phase 2 study of idelalisib in patients with refractory indolent B-cell non-Hodgkin lymphoma (iNHL). J Clin Oncol. 2014; 32:e19554.

40. Smith N, Xenakis A, Beckerman R, Chhatwal J, Gregory SA, Briggs A. A decision-analytic model of idelalisib in relapsed or refractory patients with indolent non-Hodgkin lymphoma. J Clin Oncol. 2014; 32:e17655.

41. Salles G, Schuster S, de Vos S, et al. Idelalisib efficacy and safety in follicular lymphoma patients from a phase 2 study. Paper presented at: 2015 American Society of Clinical Oncology Annual Meeting; May 28-June 3, 2015; Chicago, IL.

42. Barrientos J, Ghia P, Pagel J, et al. Outcomes of anticoagulant or antiplatelet use in patients with chronic lymphocytic leukemia or indolent non-hodgkin lymphoma in idelalisib trials. Paper presented at: ASCO Annual Meeting; May 29-June 2, 2015; Chicago, IL.

43. Coutre SE, Barrientos JC, Brown JR, et al. Management of adverse events associated with idelalisib treatment - expert panel opinion. Leuk Lymphoma. 2015;56:1-20.

44. Lampson BL, Matos T, Kim HT, et al. Idelalisib given front-line for the treatment of chronic lymphocytic leukemia results in frequent and severe immune-mediated toxicities. Blood. 2015;126(23):497.

45. O'Brien SM, Lamanna N, Kipps TJ, et al. A phase 2 study of idelalisib plus rituximab in treatment-naive older patients with chronic lymphocytic leukemia. Blood. 2015;126(25):2686-2694.

46. Imbruvica ${ }^{\circledR}$ (ibrutinib capsules) [full prescribing information]. Horsham, PA: Janssen Biotech, Inc.; 2015.

47. Flinn I, Oki Y, Patel M, et al. A phase 1 evaluation of duvelisib (IPI$145)$, a PI3K- $\delta, \gamma$ inhibitor, in patients with relapsed/refractory iNHL. Blood. 2014;124(21):abstr802.

48. Dreyling M, Cunningham D, Bouabdallah K, et al. Phase 2A study of copanlisib, a novel PI3K inhibitor, in patients with indolent lymphoma. Blood. 2014;124(21):abstract1701.

49. Barr PM, Saylors GB, Forbes Spurgeon SE, et al. Phase 2 trial of GS-9973, a selective syk inhibitor, and idelalisib (idela) in chronic lymphocytic leukemia (CLL) and non-Hodgkin lymphoma (NHL). J Clin Oncol. 2014;32(5s):abstract7059.

50. Smith SM, Pitcher B, Jung S-H, et al. Unexpected and serious toxicity observed with combined idelalisib, lenalidomide and rituximab in relapsed/refractory B cell lymphomas: Alliance A051201 and A051202. Blood. 2014;124(21):3091.

51. Cheah CY, Nastoupil LJ, Neelapu SS, Forbes SG, Oki Y, Fowler NH. Lenalidomide, idelalisib, and rituximab are unacceptably toxic in patients with relapsed/refractory indolent lymphoma. Blood. 2015; 125(21):3357-3359.

52. Zelenetz AD, Robak T, Coiffier B, et al. Idelalisib plus bendamustine and rituximab (BR) is superior to BR alone in patients with relapsed/refractory chronic lymphocytic leukemia: results of a phase 3 randomized double-blind placebo-controlled study. Blood. 2015;126(23):abstract LBA-5. 
OncoTargets and Therapy

\section{Publish your work in this journal}

OncoTargets and Therapy is an international, peer-reviewed, open access journal focusing on the pathological basis of all cancers, potential targets for therapy and treatment protocols employed to improve the management of cancer patients. The journal also focuses on the impact of management programs and new therapeutic agents and protocols on

patient perspectives such as quality of life, adherence and satisfaction. The manuscript management system is completely online and includes a very quick and fair peer-review system, which is all easy to use. Visit http://www.dovepress.com/testimonials.php to read real quotes from published authors.

Submit your manuscript here: http://www.dovepress.com/oncotargets-and-therapy-journal 